\title{
Informed consent of patients of Alzheimer's Disease
}

\author{
Hiroyuki Aizawa* \\ Aizawa Science Museum, Kasukabe, Saitama, Japan
}

Alzheimer's Disease is a specific type of amyloidosis, which develops cognitive defects caused by formation of beta-amyloids in cerebrum at the old age of more than 40. Patients with familiar Alzheimer's Disease have mutations in amyloid protein precursor gene or gamma-secretase genes, both of which encode proteins involved in production of amyloid-beta peptides, whereas patients of sporadic Alzheimer's Disease produce amyloid-beta peptides from amyloid precursor protein by dysregulated proteolytic processing under some stress such as chemical oxidation of phospholipids in bio-membranes. All through the life of patients, amyloid-beta peptides gradually and progressively form several types of aggregates such as beta-amyloid fibrils, which further develop into senile plaque at the age of more than 40. As usual in amyloidosis, beta-amyloids show some toxic effects at sites where they are formed in cerebrum.

Patients with Alzheimer's Disease gradually and progressively lose their cognitive abilities including learning, memory, decision making, language, logic, calculation, and communication, which finally collapse personality of patients. Once they lose the ability of cognition at later or severe stages of Alzheimer's Disease, it is difficult to get informed consent of subjects for clinical treatments. Accordingly, it is critical for physicians to get informed consent of patients at earlier stages of Alzheimer's disease in order to treat subjects properly and legally. This issue is quite important especially for research and development on treatment of Alzheimer's Disease including new drug discovery, which should have benefits and risks for patients. However, patients with Alzheimer's Disease have defects in cognition and thus could not understand, make decision of, and remember what physician explains about benefits and risks of clinical treatments especially at the later stages of the disease. As Alzheimer's Disease develops progressively for more than a decade, it could be possible to get informed consent before patients lose their cognitive ability if physicians were able to diagnose them at earlier stages of the disease.

Unfortunately, however, we have not yet established rigid criteria to diagnose Alzheimer's Disease especially in earlier stages. Psychological tests enable physicians to diagnose mental retardation and dementia, while it is difficult to distinguish Alzheimer's Disease at earlier stages from other dementia psychologically. At present, the most reliable technique for diagnosis of Alzheimer's Disease is the biopsy test on betaamyloid and paired helical filaments in patient's cerebrum after death. Non-invasive beta-amyloid bioimaging could not give a definitive diagnosis for Alzheimer's Disease especially at earlier stages, because

Copyright: (C2019 Aizawa H. This is an open-access article distributed under the terms of the Creative Commons Attribution License, which permits unrestricted use, distribution, and reproduction in any medium, provided the original author and source are credited. only one tenth of subjects with high content of beta-amyloids in the whole cerebrum develops Alzheimer's Disease. Discrepancy between dense accumulation of beta-amyloid all through the cerebrum and low expected ratio for development of Alzheimer's disease suggests that the cognition defects are derived from damages in some specific region of cerebrum rather than in a whole cerebrum. Technical limitation on diagnosis of Alzheimer's Disease at earlier stages makes it difficult to get informed consent of patients for treatments of the disease.

On the other hand, acetylcholine, a neurotransmitter, is well known to be down-regulated in the patients at earlier stages of the disease. Inhibition of acetylcholine esterase, which degrades acetylcholine at synapses, significantly improves cognitive defects of the subjects at early and middle stages of Alzheimer's Disease, while the inhibition could not stop progression of the disease. Furthermore, cholinergic neurons at basal nuclei of Meynert degenerate progressively in Alzheimer's Disease patients. Thus, it is quite likely that cognition defects of Alzheimer's Disease are caused by amyloidosis at this area or on their axon bundles. It is possible to use the level of acetylcholine at cerebral cortex or at basal nuclei of Meynert as a marker for diagnosis of Alzheimer's Disease at early stages of the disease.

Until now, we have few drugs for curative treatments of Alzheimer's Disease, which loses cholinergic neurons at basal nuclei of Meynert necessary for cognition. In order to develop new drugs for treatment of Alzheimer's Disease patients, it is critical to get informed consent of the patients at early stages for clinical trial. In order to diagnose at early stages of Alzheimer's disease, we need to develop novel technique probably using non-invasive bioimaging of acetylcholine together with beta-amyloids as soon as possible.

It is also important for us to think about how to respect and protect human dignity not only for individual subject but also from a point of view of social ethics. Patients should have right for making decision on acceptance or rejection of each clinical treatment at any time by their will. And even after almost entire loss of functions of cerebral cortex, patients still retain emotions and sanctity of life. We need to establish clear rule on informed consent of patients of Alzheimer's Disease not only for research and development but also for clinical treatments.

Finally, I would like to express my special thanks to all the members at Seiko Research Institute for Education, Osaka, Japan for encouragement to write essays on bioethics.

*Correspondence to: Hiroyuki Aizawa, Aizawa Science Museum, 1-10-13 Yutaka-cho, Kasukabe, Saitama 3440066, Japan, Tel: +81-48-754-9880; Fax: +8148-754-9880, E-mail: aizawa@rr.iij4u.or.jp

Received: January 02, 2019; Accepted: January 04, 2019; Published: January 07, 2019 